TRANSACTIONS OF THE

AMERICAN MATHEMATICAL SOCIETY

Volume 358, Number 5, Pages 1937-1952

S 0002-9947(05)04097-3

Article electronically published on December 20, 2005

\title{
LIMITING WEAK-TYPE BEHAVIOR FOR SINGULAR INTEGRAL AND MAXIMAL OPERATORS
}

\author{
PRABHU JANAKIRAMAN
}

\begin{abstract}
The following limit result holds for the weak-type $(1,1)$ constant of dilation-commuting singular integral operator $T$ in $\mathbb{R}^{n}$ : for $f \in L^{1}\left(\mathbb{R}^{n}\right)$, $f \geq 0$,

$$
\lim _{\lambda \rightarrow 0} \lambda m\left\{x \in \mathbb{R}^{n}:|T f(x)|>\lambda\right\}=\frac{1}{n} \int_{S^{n-1}}|\Omega(x)| d \sigma(x)\|f\|_{1} .
$$
\end{abstract}

For the maximal operator $M$, the corresponding result is

$$
\lim _{\lambda \rightarrow 0} \lambda m\left\{x \in \mathbb{R}^{n}:|M f(x)|>\lambda\right\}=\|f\|_{1} .
$$

\section{INTRODUCTION}

A dilation-commuting singular integral operator $T$ acting on $L^{p}\left(\mathbb{R}^{n}\right), 1 \leq p<\infty$, is defined by

$$
T f(x)=\lim _{\epsilon \rightarrow 0} \int_{|x-y|>\epsilon} \frac{\Omega(x-y)}{|x-y|^{n}} f(y) d y,
$$

where $\Omega$ is homogeneous of degree 0 , and satisfies the cancellation property $\int_{S^{n-1}} \Omega(x) d \sigma(x)=0$ and a continuity condition (1.3) mentioned below. In [11, the author has described the usual theory for these operators and addressed the search for the best constants in the strong-type and weak-type inequalities satisfied by these operators. The main reference is Stein [15]. The strong-type property is: For $1<p<\infty$, there exists a constant $C_{p, n}>0$ such that for all $f \in L^{p}\left(\mathbb{R}^{n}\right)$,

$$
\|T f\|_{p} \leq C_{p, n}\|f\|_{p} .
$$

The weak-type property is: For $p=1$, there exists a constant $C_{n}$ such that for all $f \in L^{1}\left(\mathbb{R}^{n}\right), \lambda>0$,

$$
m(x:|T f(x)|>\lambda) \leq \frac{C_{n}}{\lambda}\|f\|_{1} .
$$

The usual Calderón-Zygmund method of rotations gives precise information on the constant $C_{p, n}$ in (1.1) for a wide subclass of operators $T$. In fact, if $\Omega$ is an odd function, that is, if $\Omega(-x)=-\Omega(x)$, then $C_{p, n}=\frac{\pi}{2} H_{p} \int_{S^{n-1}}|\Omega(x)| d \sigma(x)$, where $H_{p}$ is the strong-type constant for the Hilbert transform. See Iwaniec-Martin [10]. Such detailed information is at present unavailable for the weak-type constant $C_{n}$ in (1.2), although a possible conjecture is that it should be $\frac{\pi}{2} H_{1} \int_{S^{n-1}}|\Omega(x)| d \sigma(x)$, where $H_{1}$ is the weak-type constant for the Hilbert transform. In [1], the author

Received by the editors September 25, 2003.

2000 Mathematics Subject Classification. Primary 42B20, $42 \mathrm{~B} 25$.

This paper is part of the author's thesis work under the direction of Professor Rodrigo Bañuelos. The research was partly supported by an NSF grant.

(C)2005 American Mathematical Society 1937 
has shown that $C_{n}$ is at worst $c \log n \int_{S^{n-1}}|\Omega(x)| d \sigma(x)$ using a certain modification of the Calderón-Zygmund decomposition and a new regularity condition on $\Omega$ : For $\xi \in S^{n-1}, 0<\delta<\frac{1}{n}$,

$$
\int_{S^{n-1}}|\Omega(x-\delta \xi)-\Omega(x)| d \sigma(x) \leq c n \delta \int_{S^{n-1}}|\Omega(x)| d \sigma(x),
$$

where $c$ is independent of dimension. The proof is then applied to the special case of the $j^{\text {th }}$ Riesz transform $R_{j}$ defined with

$$
\Omega(x)=\frac{\Gamma\left(\frac{n+1}{2}\right)}{\pi^{\frac{n+1}{2}}} \frac{x_{j}}{|x|},
$$

and its weak-type constant is shown to be at worst $c \log n$. Hence

$$
m\left(x:\left|R_{j} f(x)\right|>\lambda\right) \leq \frac{c \log n}{\lambda}\|f\|_{1} .
$$

When $n=1$, there is only one Riesz transform on the real line; this is the Hilbert transform $H$. Given $f \in L^{p}(\mathbb{R}), 1 \leq p<\infty$, define

$$
H f(x)=\frac{1}{\pi} \text { p.v. } \int_{-\infty}^{\infty} \frac{f(x-t)}{t} d t .
$$

The special feature of the Hilbert transform is that $f$ and $H f$ are the boundary values of conjugate harmonic functions on the upper half space. Let $u$ and $v$ be harmonic extensions to $\mathbb{R}_{+}^{2}$ of $f$ and $H f$, respectively. Then $u+i v$ is analytic on $\mathbb{R}_{+}^{2}$. In particular,

$$
\nabla u \cdot \nabla v=0 \text { and }|\nabla v| \leq|\nabla u| .
$$

These properties have been used to evaluate through both analytic and probabilistic techniques the exact values of the strong-type $H_{p}$ for $1<p<\infty$ and weak-type constant $H_{1}$ for $p=1$. See [13, 5] and [1]. The harmonic extensions of the Riesz transforms are also conjugate harmonic functions on the upper half space. Let $u_{j}$ be the harmonic extension of $R_{j} f$ to $\mathbb{R}_{+}^{n}$. Here $R_{0}$ is the identity operator. Then

$$
\sum_{j=0}^{n} \frac{\partial u_{j}}{\partial x_{j}}=0
$$

and for $i \neq j$,

$$
\frac{\partial u_{i}}{\partial x_{j}}=\frac{\partial u_{j}}{\partial x_{i}} .
$$

These properties have been used by several authors ([16, 8, 2, 2, 14]) to study the strong-type $(p, p)$ constant for the Riesz transform $R_{j}$, which is now known ([10, 3] ) to equal that of the Hilbert transform $H$. However the techniques used in finding the weak-type $(1,1)$ constant for $H$ ([5], [1]) rely on the gradient conditions (1.5) which do not follow from (1.6) and (1.7). Hence this question remains open for $R_{j}$.

The conjecture is that the constant in (1.4) is independent of dimension and that $C_{n}$ in (1.2) is just $c \int_{S^{n-1}}|\Omega(x)| d \sigma(x)$. Though the Calderón-Zygmund theory may not be sufficient to get this result, a modification of the decomposition followed by some precise analysis allowed the author to reduce to the $\log n$ constants stated above. By extending these methods to a limiting situation, the following theorem 
is proved in this paper. Let $\nu$ be an absolutely continuous signed measure on $\mathbb{R}^{n}$ with $|\nu|\left(\mathbb{R}^{n}\right)<\infty$. Define

$$
T \nu(x)=\lim _{\epsilon \rightarrow 0} \int_{|x-y|>\epsilon} \frac{\Omega(x-y)}{|x-y|^{n}} d \nu(y) .
$$

Here $\Omega$ is homogeneous of degree 0 , and satisfies $\int_{S^{n-1}} \Omega(x) d \sigma(x)=0$ and the regularity condition (1.3). Then

\section{Theorem 1.1.}

$$
\lim _{\lambda \rightarrow 0} \lambda m\left\{x \in \mathbb{R}^{n}:|T \nu(x)|>\lambda\right\}=\left(\frac{1}{n} \int_{S^{n-1}}|\Omega(x)| d \sigma(x)\right)\left|\nu\left(\mathbb{R}^{n}\right)\right| .
$$

The reason for working with measures is to allow some ease in writing the proof and to extend analogously to the setting of maximal operators. Note that the theorem gives the lower bound for the weak-type constant of $T$ as $\frac{1}{n} \int_{S^{n-1}}|\Omega(x)| d \sigma(x)$. Moreover it implies

\section{Corollary 1.1.}

$$
\inf \|T \nu\|_{w(1)} \geq \frac{1}{n} \int_{S^{n-1}}|\Omega(x)| d \sigma(x),
$$

where the infimum is taken over all absolutely continuous probability measures, and $\|\cdot\|_{w(1)}$ is the weak-type $(1,1)$ norm defined by

$$
\|g\|_{w(1)}=\sup _{\lambda>0} \lambda m\left\{x \in \mathbb{R}^{n}:|g(x)|>\lambda\right\} .
$$

In Section 3, analogous results are proved for the maximal operator. The HardyLittlewood maximal operator $M$ is defined by

$$
M f(x)=\sup _{r>0} \frac{1}{|B(x, r)|} \int_{B(x, r)}|f(y)| d y,
$$

for $f \in L^{p}\left(\mathbb{R}^{n}\right), 1 \leq p<\infty$. This operator is strong-type $(p, p)$ and weaktype $(1,1)$. The strong-type constant is independent of dimension $([19])$, and the question is open for the weak-type constant. Stein and Stromberg ([19]) proved that the constant $C_{n}$ in

$$
m\left\{x \in \mathbb{R}^{n}:|M f(x)|>\lambda\right\} \leq \frac{C_{n}}{\lambda}\|f\|_{1}
$$

is at worst order $n$. Following the proof of Theorem 1.1 the following limit result is proved for $M$ : for $f \in L^{1}\left(\mathbb{R}^{n}\right)$,

$$
\lim _{\lambda \rightarrow 0} \lambda m\left\{x \in \mathbb{R}^{n}: M f(x)>\lambda\right\}=\|f\|_{1} .
$$

The theorem is proved in a more general setting in Section 3, Again observe that this means

\section{Corollary 1.2.}

$$
\inf \|M f\|_{w(1)} \geq 1
$$

where the infimum is taken over all functions $f \in L^{1}\left(\mathbb{R}^{n}\right)$ with $\|f\|_{1}=1$ and $\|M f\|_{w(1)}=\sup _{\lambda>0} \lambda m\left\{x \in \mathbb{R}^{n}: M f(x)>\lambda\right\}$.

Section [5introduces these infimums as lower operator norms and suggests some open questions. 


\section{Proof of Theorem 1.1}

Let $\nu$ be an absolutely continuous signed measure on $\mathbb{R}^{n}$ with $|\nu|\left(\mathbb{R}^{n}\right)<\infty$. Define $T \nu(x)=\lim _{\epsilon \rightarrow 0} \int_{|x-y|>\epsilon} \frac{\Omega(x-y)}{|x-y|^{n}} d \nu(y)$. Here $\Omega$ is homogeneous of degree 0 , satisfies (1.3), and $\int_{S^{n-1}}|\Omega(x)| d \sigma(x)=0$. Let $\nu_{t}$ be the dilated measure: $\nu_{t}(E)=$ $\nu\left(\frac{1}{t} E\right)$. Theorem 1.1 states that

$$
\lim _{\lambda \rightarrow 0} \lambda m\left\{x \in \mathbb{R}^{n}:|T \nu(x)|>\lambda\right\}=\left(\frac{1}{n} \int_{S^{n-1}}|\Omega(x)| d \sigma(x)\right)\left|\nu\left(\mathbb{R}^{n}\right)\right| .
$$

For the proof of this, two lemmas are needed.

Lemma 2.1. $m\left\{x \in \mathbb{R}^{n}: \frac{|\Omega(x)|}{|x|^{n}}>\lambda\right\}=\frac{1}{n \lambda} \int_{S^{n-1}}|\Omega(x)| d \sigma(x)$.

Proof. Let $E_{\lambda}=\left\{x \in \mathbb{R}^{n}: \frac{|\Omega(x)|}{|x|^{n}}>\lambda\right\}$. For $\xi \in S^{n-1}$, let

$$
G_{\xi}=\left\{r \xi: 0<r<\frac{|\Omega(\xi)|^{\frac{1}{n}}}{\lambda^{\frac{1}{n}}}\right\} .
$$

Then $G_{\xi} \subset E_{\lambda}$ and $\bigcup_{\xi \in S^{n-1}} G_{\xi}=E_{\lambda}$. Therefore

$$
\begin{aligned}
m\left(E_{\lambda}\right) & =\int_{S^{n-1}} \int_{0}^{\frac{|\Omega(\xi)|^{1 / n}}{\lambda^{1 / n}}} r^{n-1} d r d \sigma(\xi) \\
& =\frac{1}{n \lambda} \int_{S^{n-1}}|\Omega(\xi)| d \sigma(\xi) .
\end{aligned}
$$

Lemma 2.2. $\lim _{t \rightarrow 0} m\left\{x \in \mathbb{R}^{n}:\left|T \nu_{t}(x)\right|>\lambda\right\}=\left(\frac{1}{n \lambda} \int_{S^{n-1}}|\Omega(x)| d \sigma(x)\right)\left|\nu\left(\mathbb{R}^{n}\right)\right|$.

Proof. Choose $\delta \ll 1, \epsilon_{1}>0, \epsilon \ll \delta \lambda$ and $t$ small so that $\left|\nu_{t}\right|\left(B\left(0, \epsilon_{t}\right)\right)>1-\epsilon$, where $\epsilon_{t} \ll \epsilon_{1}$. Let $\epsilon_{2}$ be the minimal (there is such a minimum because $|x|$ and $|x-y|$ are comparable given the placement of $x$ and $y$ ) positive real number that satisfies $\frac{1-\epsilon_{2}}{|x|^{n}} \leq \frac{1}{|x-y|^{n}} \leq \frac{1+\epsilon_{2}}{|x|^{n}}$ for all $x \in B\left(0, \epsilon_{1}\right)^{c}, y \in B\left(0, \epsilon_{t}\right)$. Note that $\epsilon_{2} \rightarrow 0$ as $t \rightarrow 0$.

Define $\nu_{t}^{1}(x)=\left.\nu_{t}\right|_{B\left(0, \epsilon_{t}\right)}$ and $\nu_{t}^{2}=\left.\nu_{t}\right|_{B\left(0, \epsilon_{t}\right)^{c}}$. Then

$$
T \nu_{t}(x)=T \nu_{t}^{1}(x)+T \nu_{t}^{2}(x) .
$$

Let

and

$$
\begin{aligned}
E_{\lambda}^{t} & =\left\{x \in \mathbb{R}^{n}:\left|T \nu_{t}(x)\right|>\lambda\right\}, \\
E_{1, \lambda}^{t} & =\left\{x \in \mathbb{R}^{n}:\left|T \nu_{t}^{1}(x)\right|>\lambda\right\},
\end{aligned}
$$

By the weak-type inequality,

$$
E_{2, \lambda}^{t}=\left\{x \in \mathbb{R}^{n}:\left|T \nu_{t}^{2}(x)\right|>\lambda\right\} .
$$

$$
\begin{aligned}
m\left(E_{2, \delta \lambda}^{t}\right) & =m\left\{x \in \mathbb{R}^{n}:\left|T \nu_{t}^{2}(x)\right|>\delta \lambda\right\} \\
& \leq \frac{C_{n}}{\delta \lambda}\left|\nu_{t}^{2}\right|\left(\mathbb{R}^{n}\right)=\frac{C_{n}}{\delta \lambda}\left|\nu_{t}\right|\left(B\left(0, \epsilon_{1}\right)^{c}\right) \\
& <\frac{C_{n} \epsilon}{\delta \lambda} .
\end{aligned}
$$

Since $E_{1,(1+\delta) \lambda}^{t} \subset E_{2, \delta \lambda}^{t} \cup E_{\lambda}^{t}$ and $E_{\lambda}^{t} \subset E_{2, \delta \lambda}^{t} \cup E_{1,(1-\delta) \lambda}^{t}$, the following estimate holds:

$$
-\frac{C_{n} \epsilon}{\delta \lambda}+m\left(E_{1,(1+\delta) \lambda}^{t}\right) \leq m\left(E_{\lambda}^{t}\right) \leq \frac{C_{n} \epsilon}{\delta \lambda}+m\left(E_{1,(1-\delta) \lambda}^{t}\right) .
$$


Since $\epsilon \ll \delta \lambda$ and $\delta \ll 1$, estimates on $m\left(E_{1, \lambda}^{t}\right)$ should approximate for $m\left(E_{\lambda}^{t}\right)$. First observe that

$$
m\left(E_{1, \lambda}^{t}\right)-\gamma_{n} \epsilon_{1}^{n} \leq m\left(E_{1, \lambda}^{t} \cap B\left(0, \epsilon_{1}\right)^{c}\right) \leq m\left(E_{1, \lambda}^{t}\right) .
$$

To estimate $m\left(E_{1, \lambda}^{t} \cap B\left(0, \epsilon_{1}\right)^{c}\right)$, split $T \nu_{t}^{1}$ into two integrals:

$T \nu_{t}^{1}(x)=\lim _{\epsilon^{\prime} \rightarrow 0} \int_{\left\{|x-y|>\epsilon^{\prime}\right\}} \frac{\Omega(x)}{|x-y|^{n}} d \nu_{t}^{1}(y)+\lim _{\epsilon^{\prime} \rightarrow 0} \int_{\left\{|x-y|>\epsilon^{\prime}\right\}} \frac{\Omega(x-y)-\Omega(x)}{|x-y|^{n}} d \nu_{t}^{1}(y)$.

For fixed $\epsilon^{\prime}$, the triangle inequality gives

$$
\begin{aligned}
& || \int_{\left\{|x-y|>\epsilon^{\prime}\right\}} \frac{\Omega(x)}{|x-y|^{n}} d \nu_{t}^{1}(y)|-| \int_{\left\{|x-y|>\epsilon^{\prime}\right\}} \frac{\Omega(x-y)-\Omega(x)}{|x-y|^{n}} d \nu_{t}^{1}(y)|| \\
\leq & \left|\int_{\left\{|x-y|>\epsilon^{\prime}\right\}} \frac{\Omega(x-y)}{|x-y|^{n}} d \nu_{t}^{1}(y)\right| \\
\leq & \left|\int_{\left\{|x-y|>\epsilon^{\prime}\right\}} \frac{\Omega(x)}{|x-y|^{n}} d \nu_{t}^{1}(y)\right|+\int_{\left\{|x-y|>\epsilon^{\prime}\right\}} \frac{|\Omega(x-y)-\Omega(x)|}{|x-y|^{n}} d\left|\nu_{t}^{1}\right|(y) .
\end{aligned}
$$

Let

$$
F_{t}=\left\{x \in B\left(0, \epsilon_{1}\right)^{c}: \lim _{\epsilon^{\prime} \rightarrow 0}\left|\int_{\left\{|x-y|>\epsilon^{\prime}\right\}} \frac{|\Omega(x-y)-\Omega(x)|}{|x-y|^{n}} d\right| \nu_{t}^{1}|(y)| \geq \delta \lambda\right\} .
$$

$F_{t}$ is estimated using the fact that $|x-y|^{n}$ and $|x|^{n}$ are comparable via the constant $\epsilon_{2}$ (see the first few lines of the proof) for $x \in B\left(0, \epsilon_{1}\right)^{c}$ and $y \in B\left(0, \epsilon_{t}\right)$ for $t$ small and the condition (1.3) assumed for the singular integral operator $T$. Also used is the fact that $\nu_{t}^{1}$ is supported in $B\left(0, \epsilon_{t}\right)$.

$$
\begin{aligned}
m\left(F_{t}\right) & \leq m\left(\left\{x \in B\left(0, \epsilon_{1}\right)^{c}: \int_{\mathbb{R}^{n}} \frac{|\Omega(x-y)-\Omega(x)|}{|x|^{n}} d\left|\nu_{t}^{1}\right|(y) \geq \frac{\delta \lambda}{\left(1+\epsilon_{2}\right)}\right\}\right) \\
& \leq \frac{1+\epsilon_{2}}{\delta \lambda} \int_{\mathbb{R}^{n}} \int_{B\left(0, \epsilon_{1}\right)^{c}} \frac{|\Omega(x-y)-\Omega(x)|}{|x|^{n}} d x d\left|\nu_{t}^{1}\right|(y) \\
& =\frac{1+\epsilon_{2}}{\delta \lambda} \int_{\mathbb{R}^{n}}\left[\int_{\epsilon_{1}}^{\infty} \frac{1}{r^{n}} \int_{S^{n-1}}\left|\Omega\left(\frac{x}{|x|}-\frac{y}{|x|}\right)-\Omega\left(\frac{x}{|x|}\right)\right|\right. \\
& \leq \frac{C_{n}\left(1+\epsilon_{2}\right)}{\delta \lambda} \int_{\mathbb{R}^{n}} \int_{\epsilon_{1}}^{\infty} \frac{|y|}{r^{2}} d r d\left|\nu_{t}^{1}\right|(y) \quad \text { by (1.3) } \\
& =\frac{C_{n}\left(1+\epsilon_{2}\right)}{\delta \lambda \epsilon_{1}} \int_{\mathbb{R}^{n}}|y| d\left|\nu_{t}^{1}\right|(y) \\
& \leq \frac{C_{n}\left(1+\epsilon_{2}\right)}{\delta \lambda \epsilon_{1}} \epsilon_{t}\left|\nu_{t}^{1}\right|\left(\mathbb{R}^{n}\right) \\
& <\frac{C_{n}\left(1+\epsilon_{2}\right) \epsilon_{t}}{\delta \lambda \epsilon_{1}} .
\end{aligned}
$$

Therefore

$$
m\left(F_{t}\right)<\frac{C_{n}\left(1+\epsilon_{2}\right) \epsilon_{t}}{\delta \lambda \epsilon_{1}}
$$


and

$$
\begin{aligned}
& m\left\{x \in B\left(0, \epsilon_{1}\right)^{c} \cap F_{t}^{c}:\left|T \nu_{t}^{1}(x)\right|>\lambda\right\} \\
\leq & m\left(E_{1, \lambda}^{t} \cap B\left(0, \epsilon_{1}\right)^{c}\right) \\
< & m\left\{x \in B\left(0, \epsilon_{1}\right)^{c} \cap F_{t}^{c}:\left|T \nu_{t}^{1}(x)\right|>\lambda\right\}+\frac{C_{n}\left(1+\epsilon_{2}\right) \epsilon_{t}}{\delta \lambda \epsilon_{1}} .
\end{aligned}
$$

On $B\left(0, \epsilon_{1}\right)^{c} \cap F_{t}^{c},(2.3)$ can be replaced by

$$
\begin{aligned}
& \lim _{\epsilon^{\prime} \rightarrow 0}\left|\int_{\left\{|x-y|>\epsilon^{\prime}\right\}} \frac{\Omega(x)}{|x-y|^{n}} d \nu_{t}^{1}(y)\right|-\delta \lambda \\
\leq & \lim _{\epsilon^{\prime} \rightarrow 0}\left|\int_{\left\{|x-y|>\epsilon^{\prime}\right\}} \frac{\Omega(x-y)}{|x-y|^{n}} d \nu_{t}^{1}(y)\right| \\
\leq & \lim _{\epsilon^{\prime} \rightarrow 0}\left|\int_{\left\{|x-y|>\epsilon^{\prime}\right\}} \frac{\Omega(x)}{|x-y|^{n}} d \nu_{t}^{1}(y)\right|+\delta \lambda .
\end{aligned}
$$

By the definition of $\epsilon_{2}, \frac{1-\epsilon_{2}}{|x|^{n}} \leq \frac{1}{|x-y|^{n}} \leq \frac{1+\epsilon_{2}}{|x|^{n}}$ for $x \in B\left(0, \epsilon_{1}\right)^{c} \cap F_{t}^{c}$ and $y \in B\left(0, \epsilon_{t}\right)$.

Therefore

$$
\begin{aligned}
& \left(1-\epsilon_{2}\right) \frac{|\Omega(x)|}{|x|^{n}}\left|\nu_{t}^{1}\left(\mathbb{R}^{n}\right)\right|-\delta \lambda \\
\leq & \lim _{\epsilon^{\prime} \rightarrow 0}\left|\int_{\left\{|x-y|>\epsilon^{\prime}\right\}} \frac{\Omega(x-y)}{|x-y|^{n}} d \nu_{t}^{1}(y)\right|=\left|T \nu_{t}^{1}(x)\right| \\
\leq & \left(1+\epsilon_{2}\right) \frac{|\Omega(x)|}{|x|^{n}}\left|\nu_{t}^{1}\left(\mathbb{R}^{n}\right)\right|+\delta \lambda .
\end{aligned}
$$

From this it follows that

$$
\begin{aligned}
\{x \in & \left.B\left(0, \epsilon_{1}\right)^{c} \cap F_{t}^{c}:\left|T \nu_{t}^{1}(x)\right|>\lambda\right\} \\
& \subset\left\{x \in B\left(0, \epsilon_{1}\right)^{c} \cap F_{t}^{c}: \frac{|\Omega(x)|}{|x|^{n}}\left|\nu_{t}^{1}\left(\mathbb{R}^{n}\right)\right|>\frac{(1-\delta) \lambda}{\left(1+\epsilon_{2}\right)}\right\}
\end{aligned}
$$

and

$$
\begin{aligned}
&\{x \in\left.B\left(0, \epsilon_{1}\right)^{c} \cap F_{t}^{c}: \frac{|\Omega(x)|}{|x|^{n}}\left|\nu_{t}^{1}\left(\mathbb{R}^{n}\right)\right|>\frac{(1+\delta) \lambda}{\left(1-\epsilon_{2}\right)}\right\} \\
& \subset\left\{x \in B\left(0, \epsilon_{1}\right)^{c} \cap F_{t}^{c}:\left|T \nu_{t}^{1}(x)\right|>\lambda\right\} .
\end{aligned}
$$

Lemma 2.1 and (2.4) can now be applied to (2.6) and (2.7) to obtain

$$
\begin{aligned}
& \frac{1-\epsilon_{2}}{n(1+\delta) \lambda} \int_{S^{n-1}}|\Omega(x)| d \sigma(x)\left|\nu_{t}^{1}\left(\mathbb{R}^{n}\right)\right|-\gamma_{n} \epsilon_{1}^{n}-\frac{C_{n}\left(1+\epsilon_{2}\right) \epsilon_{t}}{\delta \lambda \epsilon_{1}} \\
\leq & m\left\{x \in B\left(0, \epsilon_{1}\right)^{c} \cap F_{t}^{c}:\left|T \nu_{t}^{1}(x)\right|>\lambda\right\} \\
\leq & \frac{1+\epsilon_{2}}{n(1-\delta) \lambda} \int_{S^{n-1}}|\Omega(x)| d \sigma(x)\left|\nu_{t}^{1}\left(\mathbb{R}^{n}\right)\right| .
\end{aligned}
$$

Since

$$
\left|\nu\left(\mathbb{R}^{n}\right)\right|-\epsilon<\left|\nu_{t}^{1}\left(\mathbb{R}^{n}\right)\right|<\left|\nu\left(\mathbb{R}^{n}\right)\right|+\epsilon,
$$


(2.8) can be replaced by

$$
\begin{aligned}
& \frac{1-\epsilon_{2}}{n(1+\delta) \lambda} \int_{S^{n-1}}|\Omega(x)| d \sigma(x)\left|\nu\left(\mathbb{R}^{n}\right)\right|-\frac{C_{n}\left(1-\epsilon_{2}\right) \epsilon}{n(1+\delta) \lambda} \\
-\gamma_{n} \epsilon_{1}^{n}-\frac{C_{n}\left(1+\epsilon_{2}\right) \epsilon_{t}}{\delta \lambda \epsilon_{1}} & \\
\leq & m\left\{x \in B\left(0, \epsilon_{1}\right)^{c} \cap F_{t}^{c}:\left|T \nu_{t}^{1}(x)\right|>\lambda\right\} \\
\leq & \frac{1+\epsilon_{2}}{n(1-\delta) \lambda} \int_{S^{n-1}}|\Omega(x)| d \sigma(x)\left|\nu\left(\mathbb{R}^{n}\right)\right|+\frac{C_{n}\left(1+\epsilon_{2}\right) \epsilon}{n(1-\delta) \lambda} .
\end{aligned}
$$

Applying the previously found estimates gives

$$
\begin{gathered}
\frac{1-\epsilon_{2}}{n(1+\delta)^{2} \lambda} \int_{S^{n-1}}|\Omega(x)| d \sigma(x)\left|\nu\left(\mathbb{R}^{n}\right)\right|-\frac{C_{n}\left(1-\epsilon_{2}\right) \epsilon}{n(1+\delta)^{2} \lambda} \\
-\gamma_{n} \epsilon_{1}^{n}-\frac{C_{n}\left(1+\epsilon_{2}\right) \epsilon_{t}}{\delta \lambda \epsilon_{1}}-\frac{C_{n} \epsilon}{\delta \lambda} \\
\leq m\left\{x \in \mathbb{R}^{n}:\left|T \nu_{t}(x)\right|>\lambda\right\}=m\left(E_{\lambda}^{t}\right) \\
\leq \frac{1+\epsilon_{2}}{n(1-\delta)^{2} \lambda} \int_{S^{n-1}}|\Omega(x)| d \sigma(x)\left|\nu\left(\mathbb{R}^{n}\right)\right|+\frac{C_{n}\left(1+\epsilon_{2}\right) \epsilon}{n(1-\delta)^{2} \lambda} \\
+\gamma_{n} \epsilon_{1}^{n}+\frac{2 C_{n}\left(1+\epsilon_{2}\right) \epsilon_{t}}{\delta \lambda \epsilon_{1}}+\frac{C_{n} \epsilon}{\delta \lambda} .
\end{gathered}
$$

As $t \rightarrow 0, \epsilon_{t} \rightarrow 0$ and $\epsilon_{2} \rightarrow 0$. Therefore

$$
\begin{aligned}
& \frac{1}{n(1+\delta)^{2} \lambda} \int_{S^{n-1}}|\Omega(x)| d \sigma(x)\left|\nu\left(\mathbb{R}^{n}\right)\right|-\frac{C_{n}}{n(1+\delta)^{2} \lambda} \epsilon-\gamma_{n} \epsilon_{1}^{n} \\
\leq & \liminf _{t \rightarrow 0} m\left\{x \in \mathbb{R}^{n}:\left|T \nu_{t}(x)\right|>\lambda\right\} \leq \limsup _{t \rightarrow 0} m\left\{x \in \mathbb{R}^{n}:\left|T \nu_{t}(x)\right|>\lambda\right\} \\
\leq & \frac{1}{n(1-\delta)^{2} \lambda} \int_{S^{n-1}}|\Omega(x)| d \sigma(x)\left|\nu\left(\mathbb{R}^{n}\right)\right|+\frac{C_{n}}{n(1-\delta)^{2} \lambda} \epsilon+\gamma_{n} \epsilon_{1}^{n}+\frac{C_{n}}{\delta \lambda} \epsilon .
\end{aligned}
$$

Finally since $\epsilon$ is arbitrarily small relative to the remaining small constants, first let $\epsilon \rightarrow 0$ and then let $\delta \rightarrow 0$ and $\epsilon_{1} \rightarrow 0$. This gives the final result:

$$
\lim _{t \rightarrow 0} m\left\{x \in \mathbb{R}^{n}:\left|T \nu_{t}(x)\right|>\lambda\right\}=\frac{1}{n \lambda} \int_{S^{n-1}}|\Omega(x)| d \sigma(x)\left|\nu\left(\mathbb{R}^{n}\right)\right| .
$$

Proof of Theorem 1.1. Step 1: T $\nu_{t}(x)=(T \nu)_{t}(x)$

$$
\begin{aligned}
T \nu_{t}(x) & =\lim _{\epsilon \rightarrow 0} \int_{\{|y|>\epsilon\}} \frac{\Omega(x-y)}{|x-y|^{n}} d \nu_{t}(y) \\
& =\lim _{\frac{\epsilon}{t} \rightarrow 0} \int_{\left\{\frac{|y|}{t}>\frac{\epsilon}{t}\right\}} \frac{\Omega\left(\frac{x}{t}-\frac{y}{t}\right)}{t^{n}\left|\frac{x}{t}-\frac{y}{t}\right|^{n}} d \nu\left(\frac{y}{t}\right) \\
& =\frac{1}{t^{n}}(T \nu)\left(\frac{x}{t}\right)=(T \nu)_{t}(x) .
\end{aligned}
$$


Step 2:

$$
\begin{aligned}
& m\left\{x \in \mathbb{R}^{n}:\left|T \nu_{t}\right|(x)>\lambda\right\} \\
= & m\left\{x \in \mathbb{R}^{n}: \frac{1}{t^{n}}|T \nu|\left(\frac{x}{t}\right)>\lambda\right\} \\
= & m\left\{x \in \mathbb{R}^{n}:|T \nu|\left(\frac{x}{t}\right)>\lambda t^{n}\right\} \\
= & t^{n} m\left\{\frac{x}{t} \in \mathbb{R}^{n}:|T \nu|\left(\frac{x}{t}\right)>\lambda t^{n}\right\} .
\end{aligned}
$$

Therefore,

$$
\lambda m\left\{x \in \mathbb{R}^{n}:\left|T \nu_{t}\right|(x)>\lambda\right\}=\lambda t^{n} m\left\{x \in \mathbb{R}^{n}:|T \nu(x)|>\lambda t^{n}\right\} .
$$

Let $t \rightarrow 0$. Then by Lemma 2.2 ,

$$
\begin{aligned}
\frac{1}{n} \int_{S^{n-1}}|\Omega(x)| d \sigma(x) & =\lim _{t \rightarrow 0} \lambda t^{n} m\left\{x \in \mathbb{R}^{n}:|T \nu(x)|>\lambda t^{n}\right\} \\
& =\lim _{\lambda \rightarrow 0} \lambda m\left\{x \in \mathbb{R}^{n}:|T \nu(x)|>\lambda\right\} .
\end{aligned}
$$

The following is true when $\lambda \rightarrow \infty$ : For $f \in L^{1}\left(\mathbb{R}^{n}\right)$,

$$
\lim _{\lambda \rightarrow \infty} \lambda m\left\{x \in \mathbb{R}^{n}:|T f(x)|>\lambda\right\}=0 .
$$

To check this, let $\epsilon>0$ be given and let $f=f_{1}+f_{2}$, where $f_{1} \in L^{1}\left(\mathbb{R}^{n}\right) \cap L^{2}\left(\mathbb{R}^{n}\right)$, $\left\|f_{1}\right\|_{1}>(1-\epsilon)\|f\|_{1}$ and $\left\|f_{2}\right\|_{1}<2 \epsilon\|f\|_{1}$. Then

$$
\begin{aligned}
& \lambda m\left\{x \in \mathbb{R}^{n}:|T f(x)|>\lambda\right\} \\
\leq & \lambda m\left\{x \in \mathbb{R}^{n}:\left|T f_{1}(x)\right|>2^{-1} \lambda\right\}+\lambda m\left\{x \in \mathbb{R}^{n}:\left|T f_{2}(x)\right|>2^{-1} \lambda\right\} \\
\leq & \frac{C}{\lambda}\left\|f_{1}\right\|_{2}^{2}+C \epsilon\left\|f_{2}\right\|_{1} .
\end{aligned}
$$

The second inequality is due to the fact that $T$ is both weak-type $(1,1)$ and weaktype $(2,2)$. Therefore

$$
\limsup _{\lambda \rightarrow \infty} \lambda m\{x:|T f(x)|>\lambda\} \leq C \epsilon\|f\|_{1} .
$$

Since $\epsilon$ is arbitrary, the claim is proved. This is also true in the case of the maximal operators considered next.

\section{Maximal operators}

Let $\Theta=\Delta+\Sigma$ be the space of all finite signed measures on $\mathbb{R}^{n} . \Delta$ is the space of absolutely continuous measures, and $\Sigma$ is the space of mutually singular measures with respect to the Lebesgue measure $m$. Define the centered maximal operator $M$ on $\Theta$ by

$$
M \nu(x)=\sup _{r>0} \frac{|\nu|(B(x, r))}{m(B(x, r))} .
$$

Define the uncentered maximal operator $\tilde{M}$ by

$$
\tilde{M} \nu(x)=\sup _{B_{x}} \frac{|\nu|\left(B_{x}\right)}{m\left(B_{x}\right)},
$$


where the sup is taken over all balls containing $x$. Also define a difference maximal operator as follows: Let $\nu=\nu^{+}-\nu^{-}$be the Hahn decomposition for a signed measure $\nu$. Define

$$
M^{\Delta} \nu=M \nu^{+}-M \nu^{-} .
$$

Then the following limit results hold for the maximal operators. The proofs are analogous to that of Theorem 1.1 .

Theorem 3.1. Let $\nu$ be a finite signed measure on $\mathbb{R}^{n}$ :

(a) $\lim _{\lambda \rightarrow 0} \lambda m\left\{x \in \mathbb{R}^{n}: M \nu(x)>\lambda\right\}=|\nu|\left(\mathbb{R}^{n}\right)$.

(b) $\lim _{\lambda \rightarrow 0} \lambda m\left\{x \in \mathbb{R}^{n}: \tilde{M} \nu(x)>\lambda\right\}=2^{n}|\nu|\left(\mathbb{R}^{n}\right)$.

(c) $\lim _{\lambda \rightarrow 0} \lambda m\left\{x \in \mathbb{R}^{n}:\left|M^{\Delta} \nu(x)\right|>\lambda\right\}=\left|\nu\left(\mathbb{R}^{n}\right)\right|$.

The proof of part (a) requires the following analogue of Lemma 2.2. Without loss of generality, assume $\nu$ is a probability measure, that is, $\nu\left(\mathbb{R}^{n}\right)=|\nu|\left(\mathbb{R}^{n}\right)=1$.

Lemma 3.1. $\lim _{t \rightarrow 0} \lambda m\left\{x \in \mathbb{R}^{n}: M \nu_{t}(x)>\lambda\right\}=1$.

Note that the right-hand side may be replaced with $\lambda m\left\{x \in \mathbb{R}^{n}: M \delta_{0}(x)>\lambda\right\}$, where $\delta_{0}$ is the delta measure with unit mass at 0 .

Proof of Lemma 3.1. Recall $\nu_{t}(E)=\nu\left(\frac{1}{t} E\right)$. Let $\epsilon, \epsilon_{1}$ and $\delta$ be positive constants, where $\epsilon$ is small relative to $\epsilon_{1}$ and $\delta$. Choose $t$ small and corresponding $\epsilon_{t}$ such that $\nu_{t}\left(B\left(0, \epsilon_{t}\right)\right)>1-\epsilon$ and $\epsilon_{t}<\frac{\epsilon_{1}}{2}$. Note that $\epsilon_{t} \rightarrow 0$ as $t \rightarrow 0$.

Let $x \in B\left(0, \epsilon_{1}\right)^{c}$. Then $|x|>\epsilon_{1}>2 \epsilon_{t}$. Let

$$
E_{1, \lambda}^{t}=\left\{x \in B\left(0, \epsilon_{1}\right)^{c}: \lambda<M \nu_{t}(x) \leq \frac{\nu_{t}\left(\mathbb{R}^{n}\right)}{m\left(B\left(0,|x|-\epsilon_{t}\right)\right)}\right\}
$$

and

$$
E_{2, \lambda}^{t}=\left\{x \in B\left(0, \epsilon_{1}\right)^{c}: \lambda<M \nu_{t}(x) \text { and } \frac{\nu_{t}\left(\mathbb{R}^{n}\right)}{m\left(B\left(0,|x|-\epsilon_{t}\right)\right)}<M \nu_{t}(x)\right\} .
$$

Then $E_{1, \lambda}^{t}$ and $E_{2, \lambda}^{t}$ are disjoint sets whose union is

$$
\left\{x \in B\left(0, \epsilon_{1}\right)^{c}: M \nu_{t}(x)>\lambda\right\} .
$$

For $x \in E_{2, \lambda}^{t}$, there exists an $r<|x|-\epsilon_{t}$ such that

$$
\lambda<\frac{\nu_{t}(B(x, r))}{m(B(x, r))}
$$

and hence

$$
m(B(x, r))<\frac{\nu_{t}(B(x, r))}{\lambda} .
$$

By the Wiener Covering Lemma, there exists disjoint collection of such balls $B_{i}=B\left(x_{i}, r_{i}\right)$ such that $E_{2, \lambda}^{t} \subset \bigcup_{i} 5 B_{i}$. Therefore

$$
m\left(E_{2, \lambda}^{t}\right) \leq 5^{n} \sum_{i} m\left(B_{i}\right)<5^{n} \sum_{i} \frac{\nu_{t}\left(B_{i}\right)}{\lambda}<\frac{5^{n} \epsilon}{\lambda},
$$

since $B_{i} \cap B\left(0, \epsilon_{t}\right)=\emptyset$ for each $i$.

For $x \in E_{1, \lambda}^{t}$ and $R>0$,

$$
\frac{\nu_{t}(B(x, R))}{m(B(x, R))} \leq M \nu_{t}(x) \leq \frac{\nu_{t}\left(\mathbb{R}^{n}\right)}{m\left(B\left(|x|,|x|-\epsilon_{t}\right)\right)} .
$$


Let $R=|x|+\epsilon_{t}$. Then

$$
\frac{1-\epsilon}{m\left(B\left(x,|x|+\epsilon_{t}\right)\right)} \leq \frac{\nu_{t}\left(B\left(x,|x|+\epsilon_{t}\right)\right)}{m\left(B\left(x,|x|+\epsilon_{t}\right)\right)} \leq M \nu_{t}(x) \leq \frac{1}{m\left(B\left(x,|x|-\epsilon_{t}\right)\right)} .
$$

$M \delta_{0}$ also falls within these bounds. Since

$$
\frac{1}{\left(|x|-\epsilon_{t}\right)^{n}}-\frac{1}{\left(|x|+\epsilon_{t}\right)^{n}} \rightarrow 0 \text { as } t \rightarrow 0
$$

uniformly on $\mathbb{R}^{n} \backslash B\left(0, \epsilon_{1}\right)$ and

$$
\frac{\epsilon}{\left(|x|+\epsilon_{t}\right)^{n}}-\frac{\epsilon}{|x|^{n}} \rightarrow 0 \text { as } t \rightarrow 0
$$

uniformly on $\mathbb{R}^{n} \backslash B\left(0, \epsilon_{1}\right)$, it follows that for $t$ small and $x \in E_{1, \lambda}^{t}$,

$$
\begin{aligned}
\left|M \nu_{t}(x)-M \delta_{0}(x)\right| & <\delta+\frac{\epsilon}{m(B(0,|x|))} \\
& <\delta+\frac{\epsilon}{m\left(B\left(0, \epsilon_{1}\right)\right)}<2 \delta
\end{aligned}
$$

( $\delta>0$ is the prefixed constant mentioned at the start of the proof). Therefore for $x \in E_{1, \lambda}^{t}$,

$$
M \nu_{t}(x)>\lambda \text { implies } M \delta_{0}(x)>\lambda-2 \delta .
$$

Now suppose for $x \in B\left(0, \epsilon_{1}\right)^{c}, M \delta_{0}(x)>\lambda+2 \delta$. Then

$$
\frac{1}{m(B(x,|x|))}>\lambda+2 \delta \text { and }|x|<\frac{1}{\left(\gamma_{n}(\lambda+2 \delta)\right)^{\frac{1}{n}}} .
$$

For such $x$, with $t$ and $\epsilon$ chosen sufficiently small,

$$
M \nu_{t}(x)>\frac{1-\epsilon}{\gamma_{n}\left(|x|+\epsilon_{t}\right)^{n}} \geq \frac{1-\epsilon}{\gamma_{n}\left(\frac{1}{\left(\gamma_{n}(\lambda+2 \delta)\right)^{\frac{1}{n}}}+\epsilon_{t}\right)^{n}}>\lambda .
$$

Therefore in $B\left(0, \epsilon_{1}\right)^{c}$,

$$
M \delta_{0}(x)>\lambda+2 \delta \text { implies } M \nu_{t}(x)>\lambda .
$$

That is, $x \in E_{1, \lambda}^{t}$. From (3.4) and (3.5), it follows that for $\delta>0$ and $t$ small enough,

$$
m\left(M \delta_{0}>\lambda+2 \delta\right)-m\left(B\left(0, \epsilon_{1}\right)\right) \leq m\left(E_{1, \lambda}^{t}\right) \leq m\left(M \delta_{0}>\lambda-2 \delta\right),
$$

or equivalently

$$
\frac{1}{\lambda+2 \delta}-\gamma_{n} \epsilon_{1}^{n} \leq m\left(E_{1, \lambda}^{t}\right) \leq \frac{1}{\lambda-2 \delta} .
$$

Adding $m\left(E_{2, \lambda}^{t}\right)$ to the above and applying the estimate $m\left(E_{2, \lambda}^{t}\right) \leq \frac{5^{n} \epsilon}{\lambda}$ results in

$$
\frac{1}{\lambda+2 \delta}-\gamma_{n} \epsilon_{1}^{n} \leq m\left(\left\{M \nu_{t}>\lambda\right\} \cap B\left(0, \epsilon_{1}\right)^{c}\right) \leq \frac{1}{\lambda-2 \delta}+\frac{5^{n} \epsilon}{\lambda} .
$$

Now let $t \rightarrow 0$ to get liminf and limsup in the interior. Next let $\epsilon \rightarrow 0$, followed by the remaining two constants, to get the final result. 
Proof of Theorem 3.1(a). Step 1: $M \nu_{t}(x)=(M \nu)_{t}(x)$.

$$
\begin{aligned}
M \nu_{t}(x) & =\sup _{r>0} \frac{\nu_{t}(B(x, r))}{m(B(x, r))} \\
& =\sup _{r>0} \frac{\nu\left(\frac{1}{t} B(x, r)\right)}{m(B(x, r))} \\
& =\sup _{r>0} \frac{\nu\left(B\left(\frac{x}{t}, \frac{r}{t}\right)\right)}{m(B(x, r))} \\
& =\sup _{r>0} \frac{\nu\left(B\left(\frac{x}{t}, \frac{r}{t}\right)\right)}{t^{n} m\left(B\left(\frac{x}{t}, \frac{r}{t}\right)\right)} \\
& =(M \nu)_{t}(x) .
\end{aligned}
$$

Step 2: $m\left\{x \in \mathbb{R}^{n}: M \nu_{t}(x)>\lambda\right\}=t^{n} m\left\{x \in \mathbb{R}^{n}: M \nu(x)>\lambda t^{n}\right\}$.

By Step $1, M \nu_{t}(x)>\lambda$ if and only if $M \nu\left(\frac{x}{t}\right)>\lambda t^{n}$. Therefore $x \in\left\{x \in \mathbb{R}^{n}\right.$ : $\left.M \nu_{t}(x)>\lambda\right\}$ if and only if $\frac{x}{t} \in\left\{x \in \mathbb{R}^{n}: M \nu(x)>\lambda t^{n}\right\}$. This implies

$$
\frac{1}{t}\left\{x \in \mathbb{R}^{n}: M \nu_{t}(x)>\lambda\right\}=\left\{x \in \mathbb{R}^{n}: M \nu(x)>\lambda t^{n}\right\} .
$$

Taking the measure of the sets gives

$$
\frac{1}{t^{n}} m\left\{x \in \mathbb{R}^{n}: M \nu_{t}(x)>\lambda\right\}=m\left\{x \in \mathbb{R}^{n}: M \nu(x)>\lambda t^{n}\right\} .
$$

Now multiply both sides by $\lambda t^{n}$ and let $t \rightarrow 0$. Lemma 3.1 then implies the result.

Proof of Theorem 3.1 (b). The steps are the same as for part (a) with $\tilde{M}$ substituted for $M$. Since $m\left\{x \in \mathbb{R}^{n}: \tilde{M} \delta_{0}(x)>\lambda\right\}=\frac{2^{n}}{\lambda}$, the theorem follows.

Proof of Theorem 3.1(c). This follows if it is shown that $M^{\Delta} \nu_{t}$ converges in measure to the function $\frac{\nu\left(\mathbb{R}^{n}\right)}{|x|^{n}}$. This is a consequence of Corollary 3.3 .

Corollary 3.1. Let $f \geq 0, \in L^{p}\left(\mathbb{R}^{n}\right), 0<p<\infty$. Then

$$
\lim _{\lambda \rightarrow 0}\left[\lambda m\left\{x \in \mathbb{R}^{n}: M\left(f^{p}\right)(x)>\lambda\right\}\right]^{\frac{1}{p}}=\|f\|_{p} .
$$

The following is a conjecture left for the reader to verify.

Given $f \in L^{1}\left(\mathbb{R}^{n}\right) \cap L^{\infty}\left(\mathbb{R}^{n}\right), \lambda>0$,

$$
\begin{aligned}
\lim _{p \rightarrow \infty} & {\left[\lambda m\left\{x \in \mathbb{R}^{n}: M\left(f^{p}\right)(x)>\lambda\right\}\right]^{\frac{1}{p}} } \\
= & \left\{\begin{array}{l}
\|f\|_{\infty} \quad \text { if } m(|f|>1)>0, \\
0 \quad \text { if }|f|<1 \text { a.e., } \\
\chi_{(0,1)}(\lambda) \text { if } m(|f|=1)>0, m(|f|>1)=0 .
\end{array}\right.
\end{aligned}
$$

Similar results depending on the support of $f$ should be available for the case $p \rightarrow 0$.

An important fact about maximal operators is that if $f, g \in L^{\infty}\left(\mathbb{R}^{n}\right)$ with $\|f\|_{\infty} \neq\|g\|_{\infty}$, then $M f$ and $M g$ are different functions. Theorem 3.1 gives the corresponding analogue for functions in $L^{1}\left(\mathbb{R}^{n}\right)$.

Corollary 3.2. Let $f, g \in L^{1}\left(\mathbb{R}^{n}\right)$ with $\|f\|_{1} \neq\|g\|_{1}$. Then $M f$ and $M g$ are different functions.

Corollary 3.3. Let $\nu$ be a probability measure. Then $M \nu_{t} \rightarrow M \delta_{0}$ in measure. 
Proof. The notation here is the same as in the proof of Theorem 3.1(a). See the beginning of the proof for details on the small constants.

Given $\delta^{\prime}>0$, let $\frac{\delta^{\prime}}{8}<\lambda<\frac{\delta^{\prime}}{4}$. Then

$$
m\left(E_{2, \lambda}^{t}\right)<\frac{8\left(5^{n} \epsilon\right)}{\delta^{\prime}}
$$

and for $t$ small,

$$
\left|M \nu_{t}(x)-M \delta_{0}(x)\right|<\delta^{\prime}
$$

for $x \in E_{1, \lambda}^{t}$.

On $\mathbb{R}^{n} \backslash\left\{E_{1, \lambda}^{t} \cup E_{2, \lambda}^{t} \cup B\left(0, \epsilon_{1}\right)\right\} \subset\left\{x \in B\left(0, \epsilon_{1}\right)^{c}: M \nu_{t}(x) \leq \frac{\delta^{\prime}}{4}\right\}$, it is shown in proof of Theorem 3.1. (a) that the constants $t$ and $\epsilon$ can be chosen small such that $M \delta_{0} \leq \frac{\delta^{\prime}}{3}$. In particular $\left|M \nu_{t}-M \delta_{0}\right|<\delta^{\prime}$ here as well. Therefore

$$
\begin{aligned}
m\left\{x \in \mathbb{R}^{n}:\left|M \nu_{t}(x)-M \delta_{0}(x)\right|>\delta^{\prime}\right\} & \leq m\left(E_{2, \lambda}^{t}\right)+m\left(B\left(0, \epsilon_{1}\right)\right) \\
& \leq \frac{8\left(5^{n} \epsilon\right)}{\delta^{\prime}}+\gamma_{n} \epsilon_{1}^{n} .
\end{aligned}
$$

Since $\epsilon$ and $\epsilon_{1}$ are arbitrary with respect to $\delta^{\prime}$, there is convergence in measure.

Corollary 3.4. The weak-type constant for $\tilde{M}$ is at best $2^{n}$.

If $x \in B_{x}$ of radius $r$, then $B_{x} \subset B(x, 2 r)$. This implies that

$$
\tilde{M} \nu \leq 2^{n} M \nu \text {. }
$$

Therefore since Stein and Strömberg [19] prove that the weak-type constant for $M$ is bounded above by $c n$, Corollary 3.4 implies that the weak-type constant for $\tilde{M}$ is between $2^{n}$ and $c n 2^{n}$.

The next corollary is on the difference maximal operator $M^{\Delta}$. Let $L_{0}^{\infty}\left(\mathbb{R}^{n}\right)$ be the space of compactly supported $L^{\infty}$ functions with integral 0 . Define

$$
M^{1}\left(\mathbb{R}^{n}\right)=\left\{\nu \text { finite signed measure }: M^{\Delta}(\nu) \in L^{1}\left(\mathbb{R}^{n}\right)\right\} .
$$

Then

Corollary 3.5. $L_{0}^{\infty}\left(\mathbb{R}^{n}\right) \subset M^{1}\left(\mathbb{R}^{n}\right)$.

Proof. Let $f \in L_{c}^{\infty}\left(\mathbb{R}^{n}\right)$. Without loss of generality, assume $\int_{\mathbb{R}^{n}}|f(x)| d x=2$ so that $\int_{\mathbb{R}^{n}} f^{+}(x) d x=\int_{\mathbb{R}^{n}} f^{-}(x) d x=1$. Since $M^{\Delta}$ commutes with dilations, assume that $\operatorname{supp}(f) \subset B(0,1)$. As $f \in L^{\infty}\left(\mathbb{R}^{n}\right), M^{\Delta} f \in L^{\infty}\left(\mathbb{R}^{n}\right)$. Therefore,

$$
\int_{B(0, n)}\left|M^{\Delta} f(x)\right| d x<\infty .
$$

Now $\left|M^{\Delta} f\right| \leq\left|M f^{+}-M \delta_{0}\right|+\left|M f^{-}-M \delta_{0}\right|$. Therefore it suffices to show that $\int_{\mathbb{R}^{n} \backslash B(0, n)}\left|M f^{+}(x)-\frac{1}{\gamma_{n}|x|^{n}}\right| d x<\infty$. Observe as in the proof of Theorem 3.1 that in $\mathbb{R}^{n} \backslash B(0, n)$,

Hence

$$
\frac{1}{\gamma_{n}(|x|+1)^{n}} \leq M f^{+}(x) \leq \frac{1}{\gamma_{n}(|x|-1)^{n}} .
$$

$$
\left|M f^{+}(x)-\frac{1}{\gamma_{n}|x|^{n}}\right| \leq \frac{1}{\gamma_{n}(|x|-1)^{n}}-\frac{1}{\gamma_{n}(|x|+1)^{n}},
$$

an integrable function outside $B(0, n)$. Therefore $M^{\Delta} f \in L^{1}\left(\mathbb{R}^{n}\right)$. 
It is easy to see that the singular measure $\delta_{x}-\delta_{-x}$ is not in $M^{1}\left(\mathbb{R}^{n}\right)$ though the total mass is 0 . There may be absolutely continuous measures of measure 0 that are not in $M^{1}\left(\mathbb{R}^{n}\right)$. The space $H^{1}\left(\mathbb{R}^{n}\right)$ (see [18]) is the normed subspace of $L^{1}\left(\mathbb{R}^{n}\right)$ containing all functions $f$ such that the Riesz transforms $R_{j} f \in L^{1}\left(\mathbb{R}^{n}\right)$. As for $M^{1}\left(\mathbb{R}^{n}\right)$, all functions in $H^{1}\left(\mathbb{R}^{n}\right)$ have integral 0 . Hence the relevant question is to ask whether $M^{1}\left(\mathbb{R}^{n}\right)=H^{1}\left(\mathbb{R}^{n}\right)$ and whether $M^{\Delta}: M^{1}\left(\mathbb{R}^{n}\right) \rightarrow L^{1}\left(\mathbb{R}^{n}\right)$ is a bounded operator. The nonsubaddivity of $M^{\Delta}$ makes this nontrivial.

\section{Maximal COnvergence}

Corollary 3.3 shows that given a positive function $f$ with $L^{1}$-norm 1 , its dilation sequence $\left(f_{\epsilon}\right)$ converges to the delta measure $\delta_{0}$ in a maximal sense, although not in $L^{1}$. This suggests the following generalization:

Definition 4.1. Maximal convergence of measures: A sequence of finite signed measures $\nu_{i}$ converges maximally to a finite signed measure $\mu$ if $M^{\Delta} \nu_{i}$ converges in measure to $M^{\Delta} \mu$.

An alternate definition would be to require $M^{\Delta}\left(\nu_{i}-\mu\right) \rightarrow 0$ in measure. Maximal convergence may be equivalent to the following:

$$
\int_{\mathbb{R}^{n}} g(x) d \nu_{i}(x) \rightarrow \int_{\mathbb{R}^{n}} g(x) d \mu(x)
$$

for all $g \in C_{c}^{\infty}\left(\mathbb{R}^{n}\right)$. This is the case if $\nu_{i}$ is a dilation sequence converging to $\delta_{0}$.

One purpose of investigating maximal convergence would be to better understand the space of all finite signed measures. Relevant are questions regarding the completeness of $\Theta$ and denseness of $L^{1}\left(\mathbb{R}^{n}\right)$ in $\Theta$ under this convergence. Since $M$ is well defined on the space of locally finite measures, the closure of $\Theta$ if it exists at all should properly contain $\Theta$. More importantly, it opens up the question of whether the operator $M^{\Delta}$ is injective over $\Theta$. Consider the centered maximal operator $M$. It is known that if $f_{1}$ and $f_{2}$ are two functions with different $L^{\infty}$ norms, then their maximal functions are different. Corollary 3.2 states that this is also true if the $L^{1}$ norms are different. The next step in this direction is to prove this for $1<p<\infty$.

\section{LOWER OPERATOR NORMS}

Let $A$ be an operator acting on $L^{p}\left(\mathbb{R}^{n}\right), 1 \leq p \leq \infty$. Define the lower strongtype $(p, p)$ norm of $A$ by

$$
\|A\|_{p}^{l}=\inf \|A f\|_{p},
$$

where the infimum is taken over all functions $f \in L^{p}\left(\mathbb{R}^{n}\right)$ with $\|f\|_{p}=1$. Denote by $\|A\|_{p}^{l+}$ the lower norm when the infimum is taken over nonnegative functions. Analogously define the lower weak-type $(p, p)$ norm of $A$ by

$$
\|A\|_{w(p)}^{l}=\inf \|A f\|_{w(p)} .
$$

The infimum is taken over all functions $f \in L^{p}\left(\mathbb{R}^{n}\right)$ with $\|f\|_{p}=1$, and $\|\cdot\|_{w(p)}$ is defined by

$$
\|g\|_{w(p)}=\sup _{\lambda>0} \lambda^{p} m\left\{x \in \mathbb{R}^{n}:|g(x)|>\lambda\right\} .
$$

Denote by $\|A\|_{w(p)}^{l+}$ the lower weak-type $(p, p)$ norm when the infimum is taken over nonnegative functions. When $A=M$, the maximal operator, the lower norm $\|M\|^{l}$. equals the positive lower norm $\|M\|^{l+}$ since the functions are always taken 
in absolute value. However the results given below reveal that when $A=T$, the singular integral operator, the compelling problem is to find $\|T\|_{p}^{l+}$ and $\|T\|_{w(p)}^{l+}$.

Corollaries 1.1 and 1.2 state that $\|T\|_{w(1)}^{l+} \geq \frac{1}{n} \int_{S^{n-1}}|\Omega(x)| d \sigma(x)$ and $\|M\|_{w(1)}^{l} \geq$ 1. The natural conjecture is that there is equality in both cases. The limiting case is attained by the delta measure $\delta_{0}$. That is, $\left\|T \delta_{0}\right\|_{w(1)}=\frac{1}{n} \int_{S^{n-1}}|\Omega(x)| d \sigma(x)$ and $\left\|M \delta_{0}\right\|_{w(1)}=1$. This is proved implicitly in the proofs of the main theorems. However as $\delta_{0}$ is a singular measure, it is not apparent whether the infimum over nonnegative $L^{1}$ functions attains these values or something strictly greater. In the special case of the Hilbert transform, the following theorem of Stein and Weiss 21. verifies that the conjecture is true. They proved that for any measurable set $E$,

$$
\lambda m\left\{x \in \mathbb{R}^{n}:\left|H\left(\chi_{E}\right)(x)\right|>\lambda\right\}=\frac{4 \lambda|E|}{e^{\pi \lambda}-e^{-\pi \lambda}} .
$$

It follows that $\left\|H\left(\chi_{E}\right)\right\|_{w(1)}=\frac{2}{\pi}|E|$, and hence $\|H\|_{w(1)}^{l} \leq \frac{2}{\pi}$. Since

$$
\frac{2}{\pi}=\frac{1}{n} \int_{S^{n-1}}|\Omega(x)| d \sigma(x)
$$

for the Hilbert transform, there is equality. Note that the function in (5.3) also gives a candidate for $\|H\|_{p}^{l+}$. Similar considerations show that the conjecture is true for the maximal operator $M$ when $n=1$. In the general case, the function $\chi_{B(0,1)}$ may satisfy the same property with $\left\|T \chi_{B(0,1)}\right\|_{w(1)}=\frac{1}{n} \int_{S^{n-1}}|\Omega(x)| d \sigma(x)$ and $\left\|M \chi_{B(0,1)}\right\|_{w(1)}=1$. In the latter case, this is achieved if it is shown that $M \chi_{B(0,1)} \leq M \delta_{0}$ pointwise.

Davis proves in [6] that for nonnegative $f \in L^{1}(\mathbb{R})$ with $\|f\|_{1}=1$, the weaktype $(1,1)$ norm $\|H f\|_{w(1)} \leq 1$, and 1 is the best upper constant possible. Hence combining the two results,

Theorem 5.1. If $f$ is a nonnegative function in $L^{1}(\mathbb{R})$ with $\|f\|_{1}=1$, then

$$
\frac{2}{\pi} \leq \sup _{\lambda>0} \lambda m\{x \in \mathbb{R}:|H f(x)| \geq \lambda\} \leq 1,
$$

and the constants are the best possible in (5.4).

Some more facts are proved below. The first is that $\|M\|_{w(p)}^{l} \leq\|M\|_{w(1)}^{l}$. To see this, observe that for $p>1$,

$$
\begin{aligned}
\sup _{\lambda>0} \lambda^{p} m\left\{x \in \mathbb{R}^{n}:|M f(x)|>\lambda\right\} & =\sup _{\lambda>0} \lambda m\left\{x \in \mathbb{R}^{n}:|M f(x)|^{p}>\lambda\right\} \\
& \leq \sup _{\lambda>0} \lambda m\left\{x \in \mathbb{R}^{n}: M\left(|f|^{p}\right)(x)>\lambda\right\},
\end{aligned}
$$

where the last inequality follows from Jensen's inequality. Now take the inf in appropriate order to get $\|M\|_{w(p)}^{l} \leq\|M\|_{w(1)}^{l}$. However a more precise theorem can be proved for the maximal operator.

Theorem 5.2. For $n>2,\|M\|_{p}^{l}=1$ for all $\frac{n}{n-2}<p \leq \infty$.

Proof. Consider $f=\chi_{B(0,1)}$, the characteristic function on the unit ball. Then $f \leq \frac{1}{|x|^{n-2}}$. The latter function, called $w$, is superharmonic in $\mathbb{R}^{n}$, hence invariant under $M$. Let $u$ be the minimal superharmonic function above $f$. Since $f \leq w$, $u(x) \leq w(x)$ (in fact, equality holds) when $|x|>1$ and $u(x)=1$ when $|x|<1$. Hence $u \in L^{p}\left(\mathbb{R}^{n}\right)$ for $p>\frac{n}{n-2}$. But $M u=u$ since $u$ is superharmonic. Therefore $\|M u\|_{p}=\|u\|_{p}$ and $\|M\|_{p}^{l}=1$. 
Fiorenza 9] observes that nonnegative functions of one or two variables invariant under $M$ must be constant. This and the above proof can be combined to show that there exists $f \in L^{p}\left(\mathbb{R}^{n}\right)$ such that $M f=f$ and $\|f\|_{p}>0$ if and only if $n \geq 3$ and $p>\frac{n}{n-2}$. This is also proved by Korry [12] using different methods. The next two theorems give explicit information about $\|T\|_{2}^{l}$ and $\|H\|_{p}^{l}$ using the corresponding theorems for their operator norms. The singular integral operator $T$ acting on $L^{2}\left(\mathbb{R}^{n}\right)$ is a Fourier multiplier operator. That is, there exists a function $m \in$ $L^{\infty}\left(\mathbb{R}^{n}\right)$ such that $\hat{T} f(\xi)=m(\xi) \hat{f}(\xi)$. A well-known theorem on such multipliers states that the $L^{2}$ norm of $T$ equals the $L^{\infty}$ norm of $m$. Hence $\|T\|_{2}=\|m\|_{\infty}$. See 20] for the details. The same method allows the computation of $\|T\|_{2}^{l}$.

Theorem 5.3. Let $T$ be a singular integral operator satisfying the hypothesis of Theorem 1.1. Let $m$ be its Fourier multiplier. Then

$$
\|T\|_{2}^{l}=\sup \left\{t \geq 0: m\left\{x \in \mathbb{R}^{n}:|m(x)|<t\right\}=0\right\} .
$$

The lower strong-type $(p, p)$ norms of the Hilbert transform $H$ are computed using the fact that $H^{2}=I$, the identity operator. Let $H_{p}=\|H\|_{p}$. This is calculated in Pichorides [13] as

$$
H_{p}= \begin{cases}\tan \left(\frac{\pi}{2 p}\right), & 1<p \leq 2 \\ \cot \left(\frac{\pi}{2 p}\right), & 2 \leq p<\infty .\end{cases}
$$

Theorem 5.4. For $1<p<\infty,\|H\|_{p}^{l}=H_{p}^{-1}$.

Proof. Since $H^{2}=I,\|f\|_{p}=\left\|H^{2} f\right\|_{p} \leq H_{p}\|H f\|_{p}$. Therefore $\|H f\|_{p} \geq H_{p}^{-1}\|f\|_{p}$ for all $f \in L^{p}\left(\mathbb{R}^{n}\right)$. Therefore $\|H\|_{p}^{l} \geq H_{p}^{-1}$. Given $\epsilon>0$, let $f$ be such that $\|H f\|_{p}>\left(H_{p}-\epsilon\right)\|f\|_{p}$. Then $\|H f\|_{p}>\left(H_{p}-\epsilon\right)\|H(H f)\|_{p}$. Therefore there exists $g=H f \in L^{p}\left(\mathbb{R}^{n}\right)$ such that $\left(H_{p}-\epsilon\right)^{-1}\|g\|_{p} \geq\|H g\|_{p}$, hence $\left(H_{p}-\epsilon\right)^{-1} \geq\|H\|_{p}^{l}$. Since $\epsilon$ is arbitrary, $H_{p}^{-1} \geq\|H\|_{p}^{l}$. Therefore $\|H\|_{p}^{l}=H_{p}^{-1}$.

It is likely that $\|R\|_{p}^{l}=H_{p}^{-1}$ for $R=\left(R_{1}, \ldots, R_{n}\right)$, the vector Riesz transform. Note that the theorem also implies $\lim _{p \rightarrow 1}\|H\|_{p}^{l}=0=\lim _{p \rightarrow \infty}\|H\|_{p}^{l}=0$, suggesting that $\|H\|_{1}^{l}=0$ and $\|H\|_{\infty}^{l}=0$.

While Theorems 5.3 and 5.4 suggest trivial or known values for $\|T\|_{p}^{l}$ and $\|T\|_{w(p)}^{l}$, Corollary 1.1 and the easy to prove fact that $\|T\|_{1}^{l+}=\infty$ foretell nontrivial and interesting results for $\|T\|_{p}^{l+}$ and $\|T\|_{w(p)}^{l+}$.

OPEN Problem: Compute the $\|\cdot\|^{l+}$ norms for $H, R_{j}, R, T$ and $M$.

\section{Resolving The WEAK-Type CONSTANT}

The weak-type $(1,1)$ constants for the singular integral operator $T$ and the maximal operator $M$ are at worst $c \log n \int_{S^{n-1}}|\Omega(x)| d \sigma(x)$ and $c n$, respectively. This is proved in [11] and [19]. They are at best $\frac{1}{n} \int_{S^{n-1}}|\Omega(x)| d \sigma(x)$ and 1 respectively, as shown in this paper. The Calderón-Zygmund method of rotations shows that the strong-type $(p, p)$ constant for singular integral operators with odd $\Omega$ is precisely $\frac{\pi}{2} H_{p} \int_{S^{n-1}}|\Omega(x)| d \sigma(x)$. Here $H_{p}$ is the strong-type $(p, p)$ constant of the Hilbert transform. From this and the results of this paper, one may conjecture that the weak-type constant is between $\frac{\pi}{2 n} H_{1} \int_{S^{n-1}}|\Omega(x)| d \sigma(x)$ and $\frac{\pi}{2} H_{1} \int_{S^{n-1}}|\Omega(x)| d \sigma(x)$, where $H_{1}$ is the weak-type $(1,1)$ constant of the Hilbert transform. 


\section{REFERENCES}

[1] A. Baernstein II, Some sharp inequalities for conjugate functions, Indiana University Mathematics Journal 27 (1978), 833-852. MR0503717 (80g:30022)

[2] R. Bañuelos, Martingale transforms and related singular integrals, Transactions of the American Mathematical Society 293 (1986), 547-564. MR0816309(87h:60095)

[3] R. Bañuelos and G. Wang, Sharp inequalities for Martingales with applications to the Beurling-Ahlfors and Riesz transforms. Duke Mathematical Journal 80, No. 3, (1995), 575600. MR1370109 (96k:60108)

[4] R. Bañuelos and G. Wang, Davis's inequality for orthogonal martingales under differential subordination, Michigan Math. J. 47 (2000), no. 1, 109-124. MR1755259 (2001g:60100)

[5] B. Davis, On the weak type $(1,1)$ inequality for conjugate functions, Proc. Amer. Math. Soc. 44 (1974), 241-249. MR0348381 (50:879)

[6] B. Davis, On the distribution of conjugate functions of nonnegative measures, Duke Math. J. 40 (1973), 695-700. MR0324297 (48:2649)

[7] J. Duoandikoetxea, Fourier Analysis, American Mathematical Society, Providence, R.I., 2001. MR1800316 (2001k:42001)

[8] J. Duoandikoetxea and J.L. Rubio de Francia, Estimations independantes de la dimension pour les transformees de Riesz, C. R. Acad. Sci. 300, Serie I (1985), 193-196. MR0780616 (86e:42028)

[9] A. Fiorenza, A note on the spherical maximal function, Rend. Accad. Sci. Fis. Mat. Napoli (4) 54 (1987), 77-83. MR1005720 (91f:42016)

[10] T. Iwaniec and G. Martin, Riesz transforms and related singular integrals, J. Reine Angew. Math. 473 (1996), 25-57. MR 1390681 (97k:42033)

[11] P. Janakiraman, Weak-type estimates for singular integrals and Riesz transform, Indiana University Mathematics Journal 53 (2004), no. 2, 533-555. MR2060044 (2005e:42048)

[12] S. Korry, Fixed points of the Hardy-Littlewood maximal operator, Collect. Math. 52 (2001), no. 3, 289-294. MR.1885223 (2003d:42032)

[13] S.K. Pichorides, On the best value of the constants in the theorems of M. Riesz, Zygmund, and Kolmogorov, Studia Math. 44 (1972), 165-179. MR0312140(47:702)

[14] G. Pisier, Riesz transforms: a simpler analytic proof of P.-A. Meyer's inequality, Séminaire de Probabilités, XXII, 485-501, Lecture Notes in Math., 1321, Springer, Berlin, 1988. MR0960544 (89m:60178)

[15] E. M. Stein, Singular Integrals and Differentiability Properties of Functions, Princeton University Press, Princeton, 1970. MR0290095 (44:7280)

[16] E.M. Stein, Some results in harmonic analysis in $\mathbb{R}^{n}$ for $n \rightarrow \infty$, Bull. Amer. Math. Soc. 9 (1983), 71-73. MR0699317 (84g:42019)

[17] E.M. Stein, Problems in harmonic analysis related to curvature and oscillatory integrals, Proceedings of the International Congress of Mathematicians, 1986, Berkeley, CA. MR 0934224 (89d:42028)

[18] E.M. Stein with assistance of T.S. Murphy, Harmonic Analysis: Real-Variable methods, Orthogonality, and Oscillatory Integrals, Princeton University Press, Princeton, 1993. MR.1232192 (95c:42002)

[19] E.M. Stein and J.-O. Strömberg, Behavior of maximal functions in $\mathbb{R}^{n}$ for large $n$, Ark. Mat. 21 (1983), no. 2, 259-269. MR0727348 (86a:42027)

[20] E.M. Stein and G. Weiss, Introduction to Fourier analysis on Euclidean spaces, Princeton University Press, Princeton, 1971. MR0304972 (46:4102)

[21] E.M. Stein and G. Weiss, An extension of a theorem of Marcinkiewicz and some of its applications, J. Math. Mech. 8 (1959), 263-284. MR0107163 (21:5888)

Department of Mathematics, Purdue University, West Lafayette, Indiana 47906

E-mail address: pjanakir@math.purdue.edu

Current address: Department of Mathematics, University of Illinois-Champaign, Urbana, Illinois 61801

E-mail address: pjanakir@math.uiuc.edu 